\title{
What We Cannot Learn From Neuroanatomy About Language Learning and Language Processing. Commentary on Uylings
}

\author{
Peter Hagoort \\ F. C. Donders Centre for Cognitive Neuroimaging, \\ Radboud University Nijmegen
}

Cognitive neuroscience aims at specifications of human cognition as instantiated in the human brain. This requires that information from multiple levels be taken into consideration and that information at one level be used to guide the investigations at another level. With regard to the human language faculty, linguistics specifies structural descriptions of the relevant knowledge types such as phonological, syntactic, and semantic structures. Psycholinguistics specifies the processing architectures of different language functions such as speaking, reading, and listening. These processing architectures explain how, in real time, the different sources of linguistic knowledge in long-term memory are recruited and exploited in mapping sound or orthography onto meaning (in listening and reading) or vice versa (in speaking and writing). Finally, (cognitive) neuroscience specifies how the processing architectures are instantiated in the human brain. In this context, an account of the neural architecture of language should specify which brain areas are recruited for different language functions, as well as how these areas communicate while these

I am grateful for the comments of Pascal Fries, Peter Indefrey, and Ivan Toni on an earlier version of my contribution.

Correspondence concerning this article should be addressed to Peter Hagoort, F. C. Donder's Centre for Cognitive Neuroimaging, P.O. Box 9101, 6500 HB Nijmegen, The Netherlands. Internet:Peter.Hagoort@fcdonders.ru.nl 
functions are executed. Classically, neuropsychological data from patients with a lesion in the language-relevant cortex have been used in studies on the neural architecture of language. The advent of a whole generation of advanced neuroimaging techniques (positron-emission tomography [PET], functional magnetic resonance imaging [fMRI], magnetic-encephalography [MEG]) in the recent decades, however, replaced lesion studies as the major tool for investigating neural architecture issues.

As everyone involved in cognitive neuroscience of language realizes, it is far from trivial to relate to each other the levels of linguistic description, processing architecture, and neural hardware. This is partly due to the differences in the conceptual vocabulary in which accounts at the distinct levels are couched. However, we also suffer from the fact that we do not have a full grasp of the mapping relations between the different levels. For instance, is the mapping between specific components of the cognitive architecture and a set of anatomically defined brain areas one-to-one, one-to-many, or many-to-one (Mehler, Morton, \& Jusczyk, 1984)? It would be even worse if there does not exist a transparent or lawful relation between cognitive architecture and neural architecture, as some have claimed (Fodor, 1975). In the latter case, the attempt to connect brain and behavior is a useless endeavor. I take the success of cognitive neuroscience in many domains of cognition as an indication that even in the absence of full knowledge about the exact nature of the mapping relation between cognitive and neural architectures, this relation is less opaque and more direct than proponents of the functional stance have claimed. Such is the general assumption underlying cognitive neuroscience research. Within this broad framework of cognitive neuroscience, one has to look in more detail what the constraints and contributions of different subdomains are in designing a full account of the neurocognition of language. In the remainder, I will focus on the constraints from neuroanatomy, a field that is discussed in this volume by Uylings.

A prime example of the contribution of neuroanatomy is the famous map by Brodmann (1869-1918). This map consists of 47 
different areas, usually referred to by expressions such as BA 44 for Brodmann Area 44. The numbers of the Brodmann Areas were determined by the order in which Brodmann went through the brain, analyzing one area after the other. Brodmann's classification is based on the cytoarchitectonics of the brain, which refers to the structure, form, and position of the cells in the six layers of the cortex. Quantification was done by Brodmann on postmortem brains. These were sectioned into slices of 5-10 $\mu \mathrm{m}$ thickness that underwent Nissl staining and were then inspected under the microscope. In this way, the distribution of different cell types across cortical layers and brain areas could be determined. Even today, Brodmann's map, which was published in 1909, is seen as a hallmark in the history of neuroscience. It was published in Leipzig by the publisher Barth after almost 10 years of hard work under the title Vergleichende Lokalisationslehre der Grosshirnrinde in ihren Prinzipien dargestellt auf Grund des Zellenbaues. Brodmann's work reveals that the composition of the six cortical layers, in terms of cell types, varies across the brain. Also, cell numbers can vary. The primary visual cortex, for instance, has about twice as many neurons per cortical column as other brain areas (Amaral, 2000).

The classical view among neuroanatomists is that these architectural differences in brain structure are indicative of functional differences and, conversely, that functional differences demand differences in architecture (Bartels \& Zeki, 2005; Brodmann, 1905; Vogt \& Vogt, 1919; Von Economo \& Koskinas, 1925). Following the classical view, through different ways of characterizing brain structure (i.e., cyto-, myelo-, and receptorarchitectonics; Zilles \& Palomero-Gallagher, 2001), brain areas can be identified, for which differences in structural characteristics imply functional differences. From this view, it follows that one should look for the structural features that determine why a particular brain area can support, for instance, the processing of a first or second language.

In contrast to the classical view in neuroanatomy, more recent accounts have argued that from a computational perspective, 
different brain areas are very similar. For instance, Douglas and Martin (2004) argued that

The same basic laminar and tangential organization of the excitatory neurons of the neocortex, the spiny neurons, is evident wherever it has been sought. The inhibitory neurons similarly show a characteristic morphology and patterns of connections throughout the cortex (...) all things considered, many crucial aspects of morphology, laminar distribution, and synaptic targets are very well conserved between areas and between species. (p. 439)

Functional differences between brain areas are in this perspective mainly due to variability of the input signals in forming functional specializations. Domain specificity of a particular piece of cortex might thus not so much be determined by heterogeneity of brain tissue, but by the way in which its functional characteristics are shaped by the input.

Recent neuroimaging studies provide support for this view. A number of remarkable forms of neural plasticity have been reported in recent years. I will here discuss one example. In a recent study by Amedi, Raz, Pianka, Malach, and Zohary (2003), the authors report that they found increased activation in the primary visual cortex (V1) during a verbal memory task in congenitally blind subjects. Moreover, the stronger the activation in V1, the better the memory performance. If the structural properties of V1 had been decisive for its functional capacities, then it would be hard to see how the same neurons that in seeing people support vision could be recruited in the blind for verbal memory. This demonstrates that the cytoarchitectonic constraints for specifications of cognitive function are rather loose. Presumably, the input and the patterns of connectivity between areas are a more relevant functional parameter than the differences in the composition of cortical layers.

With respect to the language function, comparative neuroanatomy further supports my point. In a recent study (Petrides, Cadoret, \& Mackey, 2005), an area in the macaque monkey has been described that is cytoarchitectonically comparable to BA 44 
in humans, which is part of Broca's area. BA 44 in humans has been found to be involved in syntactic processes, among other things. The homologous area in the macaque monkey subserves orofacial movements. One could make an argument that in the course of evolution, human language has developed out of a system of vocalizations that require the involvement of orofacial musculature. However, my point here is that whatever the evolutionary trajectory to language, an area in the macaque monkey with a very similar cytoarchitectonic structure as Broca's area has a clearly different function than in humans. This again supports the claim that the purely cytoarchitectonic constraints for specifications of cognitive functions do not seem to be very strong.

All of this does not imply that I deny the great importance of cytoarchitectonic structures for human cognition. Clearly, without these basic building blocks of the brain, cognition would not be possible. Without neurons, glia, and axons, the cognitive machinery would not work. However, the issue here is that these building blocks enter into processes of functional specialization. My claim is that the exact nature of these functional specializations is more easily inferred from an analysis of input and connectivity than by looking at the cytoarchitectonic characteristics.

What are the consequences for accounts of language learning and language processing? I suggest that important evidence can come from different patterns of brain activation for different aspects of language processing (e.g., syntax vs. semantics). At the same time, I do not think that at the moment there is much evidence for the claim that much hinges on whether a particular activation is found in, say, BA 44 rather than BA 45. An approach based on reversed inferences from structural anatomy to cognitive function does not seem well constrained enough in the light of our current knowledge. It is functional anatomy that counts, and that might provide stronger constraints than structural anatomy for specifications of the different forms of human cognition. This implies that we should look at patterns 
of associations and dissociations in measures of brain activity. These might provide more direct insights into the neural instantiations of cognitive functions than the classical anatomical measures.

In summary, a cognitive neuroscience approach of language takes information and constraints from different levels of analysis into consideration, in the service of a full account of the neurocognition of language. The assumption hereby is that different levels can be connected in a transparent way. At the same time, not all constraints have the same force. Here it is argued that the constraints provided by the classical anatomical measures (cytoarchitectonics and myeloarchitectonics) in our current understanding are only very loose constraints for detailed specifications of cognitive functions, including language learning and language processing. However, measures of the computational features of brain tissue might provide stronger constraints. For the time being, our best bet for understanding cognitive specialization is to focus on measures of functional rather than structural neuroanatomy.

\section{References}

Amaral, D. G. (2000). The anatomical organization of the central nervous system. In E. R. Kandel, J. H. Schwartz, \& Th. M. Jessell (Eds.), Principles of neural science (4th ed., pp. 317-336). New York: McGraw-Hill.

Amedi, A., Raz, N., Pianka, P., Malach, R., \& Zohary, E. (2003). Early “visual” cortex activation correlates with superior verbal memory performance in the blind. Nature Neuroscience, 6, 758-766.

Bartels, A., \& Zeki, S. (2005). The chronoarchitecture of the cerebral cortex. Philosophical Transactions of the Royal Society of London B, 360, 733750.

Brodmann, K. (1905). Beiträge zur histologischen Lokalisation der Grosshirnrinde. Dritte Mitteilung: Die Rindenfelder der niederen Affen. Journal of Psychology and Neurology, 4, 177-226.

Douglas, R. J., \& Martin, K. A. (2004). Neuronal circuits of the neocortex. Annual Review of Neuroscience, 27, 419-451.

Fodor, J. D. (1975). The language of thought. New York: Thomas Y. Crowall.

Mehler, J., Morton, J., \& Jusczyk, P. W. (1984). On reducing language to biology. Cognitive Neuropsychology, 1, 83-116. 
Petrides, M., Cadoret, G., \& Mackey, S. (2005). Orofacial somatomotor responses in the macaque monkey homologue of Broca's area. Nature, 435, 1235-1238.

Vogt, C., \& Vogt, O. (1919). Allgemeinere Ergebnisse unserer Hirnforschung. Vierte Mitteilung. Die physiologische Bedeutung der architektonischen Rindenfelderung auf Grund neuer Rindenreizungen. Journal für Psychologie und Neurologie, 25, 279-462.

Von Economo, C., \& Koskinas, G. N. (1925). Die Cytoarchitektonik der Hirnrinde des erwachsenen Menschen. Wien, Berlin: Springer-Verlag.

Zilles, K., \& Palomero-Gallagher, N. (2001). Cyto-, myelo-, and receptor architectonics of the human parietal cortex. Neuroimage, 14, S8-S20. 